\title{
Legendre Wavelets-Picard Iteration Method for Solution of Nonlinear Initial Value Problems
}

\author{
Fu-Kang Yin, Wang-Yi Han, Jun-Qiang Song, and Xiao-Qun Cao
}

\begin{abstract}
Numerical methods for solving initial value problems (IVPs) are of fundamental importance for analyzing and controlling dynamic systems. In this paper, we first present a new Legendre wavelets-Picard iteration method (LWPIM) for solving IVPs. Combining Legendre wavelets method with Picard iteration method, LWPIM iteratively refines estimates of the solutions until the iteration converges and can avoid the solution of a system of algebraic equations. Furthermore, the developed vector-matrix form makes MCPI methods computationally efficient. Numerical results show that the proposed method is mathematically simple and easy to implement while still keeps the high accuracy.
\end{abstract}

Index Terms-Picard iteration method, legendre wavelets, IVPS, nonlinear.

\section{INTRODUCTION}

Numerical methods for solving initial value problems (IVPs) play an important role in analyzing and controlling dynamic systems that are described by ordinary differential equations. Although there are already exists a class of methods for solving IVPs, there are still remain several challenges and therefore it's urgent to improve or develop numerical techniques or approximate approaches. Among these issues are: (i) How to find the numerical solution or approximate solution when the nonlinearities are not analytical functions of the dependent variables; and (ii) How to significantly reduce the computational burdens.

Iterative techniques for the solution of nonlinear equations usually do not require that the nonlinearities be analytical functions of the dependent variables [1]. These techniques include the well-known Picard fixed-point iterative procedure and the variational iteration method. However, J.I. Ramos [2] has point out that, for IVPs in ordinary differential equations, the variational iteration method can be derived from the well-known Picard (or fixed-point) iterative technique. What's more, Picard's iterative technique provides a convergent sequence of approximations (or iterates) to the locally unique solution if a Lipschitz-continuity condition is met. Therefore, we will choose the Picard iteration method instead of variation method as our iteration framework.

Wavelet theory is a relatively new and an emerging area in

Manuscript received September 19, 2012; revised December 23, 2012. This work is supported by National Natural Science Foundation of China (Grant Nos. 41105063).

The authors are with National University of Defense Technology, Changsha, P.R. China (e-mail: yinfukang@nudt.edu.cn, hanwangyige@163.com, caoxiaoqun@nudt.edu.cn). mathematical research. Wavelets permit the accurate representation of a variety of functions and operators. Moreover, wavelets establish a connection with fast numerical algorithms [3]. Various wavelets [4]-[8] have been use for studying problems of greater computational complexity and proved to be powerful tools to explore a new direction in solving differential equations. The fundamental idea of Legendre wavelets method is, the PDE problems can be converted into those of solving a system of algebraic equations by the operational matrices of integral or derivative, thus greatly simplify the problem and reduce the computation cost [9]. It is worthy to mention here that, the method based on using the operational matrices of an orthogonal function for solving differential equations is computer oriented.

The object of the present paper is to implement Legendre wavelets method within the framework of Picard iteration method and to facilitate the computational work of the method while still keep the accuracy in the one hand. The remainder of the paper is organized as follows. Section II we describe some basic preliminaries of Legendre wavelets and some useful corollaries for our subsequent development. In Section III, we propose a new Legendre wavelets-Picard iteration method (LWPIM). In order to demonstrate the validity and applicability of LWPIM, three examples are given in section IV. Finally, a brief summary is presented.

\section{PRELIMINARIES}

In this section, we first review the basic definitions and properties of Legendre wavelets and then introduce the block pulse functions (BPFs). Finally we apply the properties of BPFs to calculate the coefficient vector of nonlinear term expanded by Legendre wavelets.

\section{A. Legendre Wavelets}

Legendre wavelets $\psi_{n m}(t)=\psi(k, \hat{n}, m, t)$ have four arguments; $\hat{n}=2 n-1, n=1,2,3, \cdots, 2^{k-1}, k$ can assume any positive integer, $m$ is the order for Legendre polynomials and $t$ is the normalized time. They are defined on the interval $[0,1)$ as follows:

$$
\psi_{n m}(t)= \begin{cases}\sqrt{m+1 / 2} 2^{\frac{k}{2}} L_{m}\left(2^{k} t-\hat{n}\right), & \text { for } \frac{\hat{n}-1}{2^{k}} \leq t \leq \frac{\hat{n}+1}{2^{k}} \\ 0, & \text { otherwise }\end{cases}
$$

where $m=0,1,2, \cdots, M-1, n=1,2, \cdots, 2^{k-1}$.

The coefficient $\sqrt{m+1 / 2}$ is for orthonormality, the dilation parameter is $a=2^{-k}$ and the translation parameter $b=\hat{n} 2^{-k}$. 
Here, $L_{m}(t)$ are the well-known Legendre polynomials of order $m$ which are defined on the interval $[-1,1]$.

A function $f(t)$ defined over $[0,1)$ may be expanded by Legendre wavelet series as

$$
f(t)=\sum_{n=1}^{+\infty} \sum_{m=0}^{+\infty} c_{n m} \psi_{n m}(t)
$$

where

$$
c_{n m}=<f(t), \psi_{n m}(t)>
$$

in $(6),<_{., .}>$denotes the inner product.

If the infinite series in Eq. (2) is truncated, then Eq. (2) can be written as

$$
f(t)=\sum_{n=1}^{2^{k-1}} \sum_{m=0}^{M-1} c_{n m} \psi_{n m}(t)=C^{T} \Psi(t)
$$

where $C$ and $\Psi(\mathrm{t})$ are $2^{k-1} M \times 1$ matrices given by

$$
\begin{gathered}
C=\left[c_{10}, c_{11}, \cdots, c_{1 M-1}, c_{20}, \cdots, c_{2 M-1}, \cdots, c_{2^{k-1} 0}, \cdots, c_{2^{k-1} M-1}\right]^{T} \\
\Psi(t)=\left[\begin{array}{l}
\psi_{10}(t), \psi_{11}(t), \cdots, \psi_{1 M-1}(t), \cdots, \\
\psi_{2^{k-1} 0}(t), \cdots, \psi_{2^{k-1} M-1}(t)
\end{array}\right]^{T}
\end{gathered}
$$

Legendre wavelet operational matrices of derivative and integration have been derived in Ref. [4]-[9].

\section{B. Block Pulse Function}

Block pulse functions form a complete set of orthogonal functions which defined on the interval $[0, b)$ by

$$
b_{i}(t)=\left\{\begin{array}{l}
1, \quad \frac{i-1}{m} b \leq t<\frac{i}{m} b \\
0, \text { elsewhere }
\end{array}\right.
$$

for $i=1,2, \cdots, m$. It is also known that for any absolutely integrable function $f(t)$ on $[0, b)$ can be expanded in block pulse functions:

$$
f(t) \simeq \xi^{T} B_{m}(t)
$$

in which

$$
\xi^{T}=\left[f_{1}, f_{2}, \cdots, f_{m}\right], B_{m}(t)=\left[b_{1}(t), b_{2}(t), \cdots, b_{m}(t)\right],
$$

where $f_{i}$ are the coefficients of the block-pulse function, given by

$$
f_{i}=\frac{m}{b} \int_{0}^{b} f(t) b_{i}(t) d t=\frac{m}{b} \int_{\left(\frac{i-1}{m}\right) b}^{\left(\frac{i}{m}\right) b} f(t) b_{i}(t) d t
$$

Definition 1 Let $A$ and $B$ are two vectors of $m$, then $A \otimes B=\left(a_{i} \times b_{i}\right)_{m}$.

Corollary 1 Assuming $f(t)$ and $g(t)$ are two absolutely integrable functions, which can be expanded in block pulse function as $f(t)=F B(t)$ and $g(t)=G B(t)$ respectively, then we have

$$
f(t) g(t)=F B(t) B^{T}(t) G^{T}=H B(t) .
$$

where $H=F \otimes G$.

\section{Proof}

By using the disjointness property of BPFs, we have

$$
\begin{aligned}
F B(t) B^{T}(t) G^{T} & =\left[f_{1} g_{1} \phi_{1}(t), f_{2} g_{2} \phi_{2}(t), \cdots, f_{m} g_{m} \phi_{m}(t)\right] \\
& =H B(t)
\end{aligned}
$$

\section{Nonlinear Term Approximately}

The Legendre wavelets can be expanded into $m$-set of block-pulse Functions as

$$
\Psi(t)=\Phi_{m \times m} B_{m}(t) .
$$

taking the collocation points as following

$$
t_{i}=\frac{i-1 / 2}{2^{k-1} M}, i=1,2, \cdots, 2^{k-1} M
$$

The $m$-square Legendre matrix $\Phi_{m \times m}$ is defined as:

$$
\Phi_{m \times m} \triangleq\left[\begin{array}{llll}
\Psi\left(t_{1}\right) & \Psi\left(t_{2}\right) \quad \cdots \quad \Psi\left(t_{2^{k-1} M}\right)
\end{array}\right] .
$$

The operational matrix of product of Legendre wavelets can be obtained by using the properties of BPFs, let $f(t)$ and $g(t)$ are two absolutely integrable functions, which can be expanded in Legendre wavelets as $f(t)=F^{T} \Psi(t)$ and $g(t)=G^{T} \Psi(t)$ respectively.

From (12), we have

$$
\begin{aligned}
& f(t)=F^{T} \Psi(t)=F^{T} \Phi_{m m} B(t), \\
& g(t)=G^{T} \Psi(t)=G^{T} \Phi_{m m} B(t),
\end{aligned}
$$

By employing Lemma 1 and Eq. (17), we get

$$
\begin{aligned}
f(t) g(t) & =\left(F^{T} \Phi_{m m} \otimes G^{T} \Phi_{m m}\right) B(t) \\
& =\left(F^{T} \Phi_{m m} \otimes G^{T} \Phi_{m m}\right) \operatorname{inv}\left(\Phi_{m m}\right) \Phi_{m m} B(t) \\
& =\left(F^{T} \Phi_{m m} \otimes G^{T} \Phi_{m m}\right) \operatorname{inv}\left(\Phi_{m m}\right) \Psi(t)
\end{aligned}
$$

\section{LEGENDRE WAVELETS-PICARD ITERATION METHOD}

In this section, we present a Legendre wavelets-Picard iteration method. This algorithm can be implemented for solving nonlinear IVPs. 
Consider the following forms of initial value problems.

$$
\frac{d u}{d t}=f(t, u) \quad t \in[a, b]
$$

and given initial condition $u(t=a)=u_{0}$, where $t=a$ is the initial time and $t=b$ is the final time. The conditions for the existence and uniqueness of the IVP solution are the same as the conditions for the classical Picard iterations to converge.

The first step of LWPIM is to transform the generic independent variable $t$ to a new variable $\tau$, which is defined on the valid range $0 \leq \tau \leq 1$ of Legendre wavelets through

$$
t=\tau(b-a)+a
$$

After the substitution, Eq. (17) is transformed to a new form as

$$
\frac{d u}{d \tau}=(b-a) f(\tau(b-a)+a, u)=g(\tau, u) \quad \tau \in[0,1]
$$

By applying the Picard iteration method, we have

$$
u_{n+1}(\tau)=u_{0}+\int_{0}^{\tau} g\left(\varsigma, u_{n}(\varsigma)\right) d \varsigma
$$

To start the iteration, an initial guess of the solution $u_{0}(\tau)$ is required. We expanded $u_{n+1}(\tau)$ and the force function $g(\tau, u)$ by Legendre wavelets as

$$
u_{n}(\tau)=C_{n}^{T} \Psi(\tau), g(\tau, u)=G_{n}^{T} \Psi(\tau)
$$

Substituting (21) into (20) and then using the Corollary 3, we have

$$
C_{n+1}^{T}=C_{0}^{T}+G_{n}^{T} P
$$

The vector $G_{n}^{T}$ can be obtained by the method described in the Section II. Although the LWPIM only converges on a finite interval, we can apply a piecewise approach to solve a significant family of IVPs over a large domain. The initial conditions on the next segment are the final state values on the previous segment.

\section{NUMERICAL EXAMPLES}

In order to assess the effectiveness and the accuracy of the proposed method, it is applied to three examples.

Example 1. Consider following nonlinear equation:

$$
u_{t}+u^{2}=0, \quad t \in[0,2]
$$

with the initial condition $u(0)=1$ and the exact solution $u(t)=1 /(1+t)$.

According to the LWPIM, we have

$$
u_{n}(t)=C_{n}^{T} \Psi(t), N\left[u_{n}(t)\right]=-u_{n}^{2}=G_{n}^{T} \Psi(t)
$$

and then we can construct the following iteration formula

$$
C_{n+1}^{T}=C_{0}^{T}+G_{n}^{T} P
$$
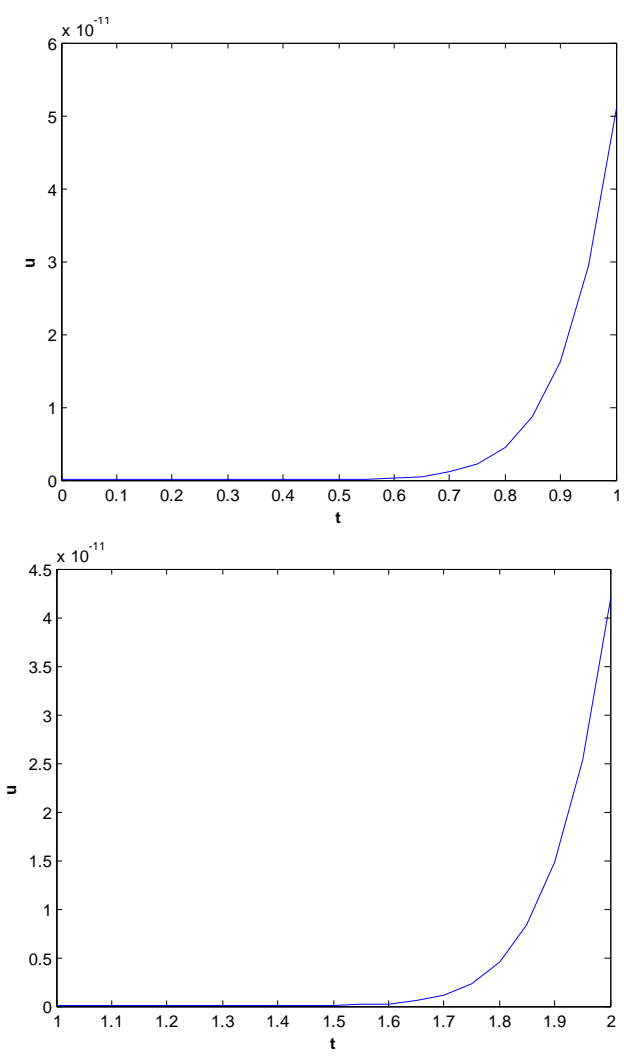

Fig. 1. Relatively errors between the LWPIM and the exact solution of Exp.1.

Fig. 1 shows the relatively errors between the LWPIM and the exact solution. We let stopping criterion $S C=1.0 e^{-10}$, $k=1$ and $M=20$. From the graphical results in the Fig. 1, it is clear that the approximate solutions converge to the exact solution.

Example 2. The Painlevé equations were first derived, during 1895-1910, in the investigations by Paul Painlevé and B.Gambier. Second painlevé equation is defined as follows:

$$
\frac{d^{2} u}{d x^{2}}=6 u^{2}(x)+x, x \in[0,1)
$$

with the initial conditions given by:

$$
u(0)=1, u^{\prime}(0)=0 \text {. }
$$

The solution of the second Painlevé equation has investigated by means of Adomian decomposition method, homotopy perturbation method, Legendre tau method and VIM [10]-[12].

In order to use LWPI method, we covert Eq. (38) into a system of equations as

$$
\left\{\begin{array}{l}
\frac{d u}{d x}=v \\
\frac{d v}{d x}=6 u^{2}(x)+x
\end{array}\right.
$$


with the initial condition $u(0)=1, v(0)=0$.

We assume $u_{n}(x)$ and $v_{n}(x)$ can be expanded by the Legendre wavelets

$$
\begin{aligned}
& u_{n}(x)=C_{n}^{T} \Psi(x), v_{n}(x)=B_{n}^{T} \Psi(x), \\
& N\left[u_{n}\right]=6 u_{n}^{2}+x=G_{n}^{T} \Psi(x)
\end{aligned}
$$

According to the LWPIM, we can construct the following iteration formula:

$$
\begin{aligned}
& C_{n+1}^{T}=C_{0}^{T}+B_{n}^{T} P \\
& B_{n+1}^{T}=B_{0}^{T}+G_{n}^{T} P
\end{aligned}
$$

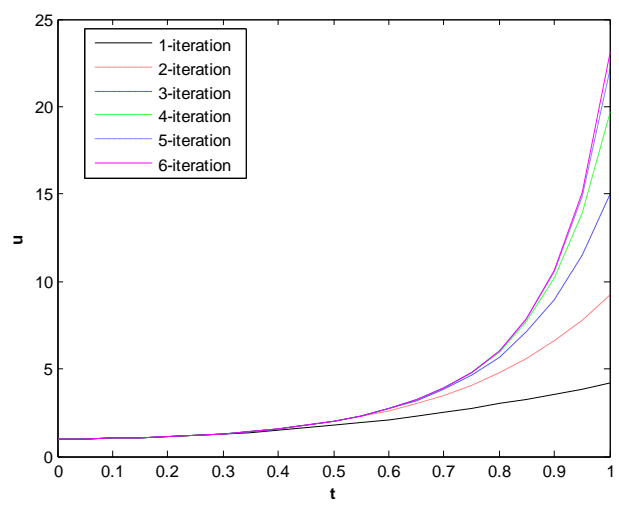

Fig. 2. Results of LWPIM.

We also give the approximately solutions of LWPIM in Fig. 2. From the first six iteration solutions of LWPIM, we can find that the solutions of LWPIM will converge after a number of iterations.

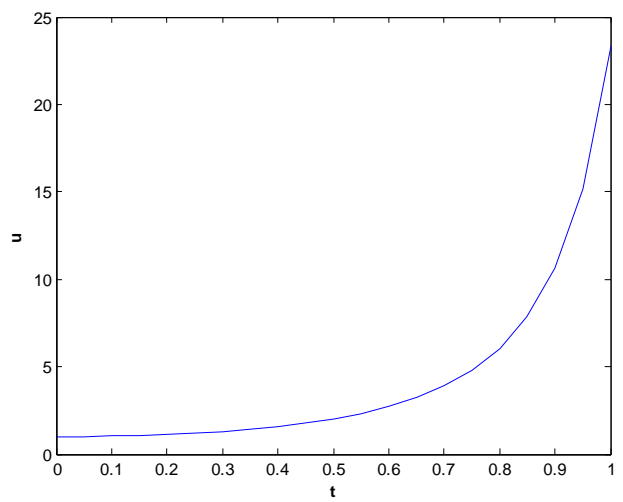

Fig. 3. Relatively errors between the LWPIM and the exact solution of Exp.2.

Fig. 3 gives the solution of LWPIM when $S C=1.0 e^{-10}$, $k=1$ and $M=24$. LWPIM iterates 30 times and the error between the last two iterations is $5.12 e^{-11}$.

Example 3. The Riccati equations are one of the most important classes of nonlinear differential equations and play a significant role in many fields of applied science [13]. The importance of the Riccati equation usually arises in optimal control problems. Consider the following Riccati equation[14], [15]

$$
\frac{d u(t)}{d t}=2 u(t)-u^{2}(t)+1, u(0)=0, t \in[0,2]
$$

with the initial condition is $u(0)=0$, which has the solution

$$
u(t)=1+\sqrt{2} \tanh \left[\sqrt{2} t+\frac{1}{2} \log \left(\frac{\sqrt{2}-1}{\sqrt{2}+1}\right)\right]
$$

Fig. 4 gives the solution of LWPI method when $S C=1.0 e^{-11}$, $k=1$ and $M=24$. LWPI method iterates 12 times and the error between the last two iterations is $3.889 e^{-12}$. From the graphical results in the Fig. 1, it is clear that the approximate solutions converge to the exact solution.
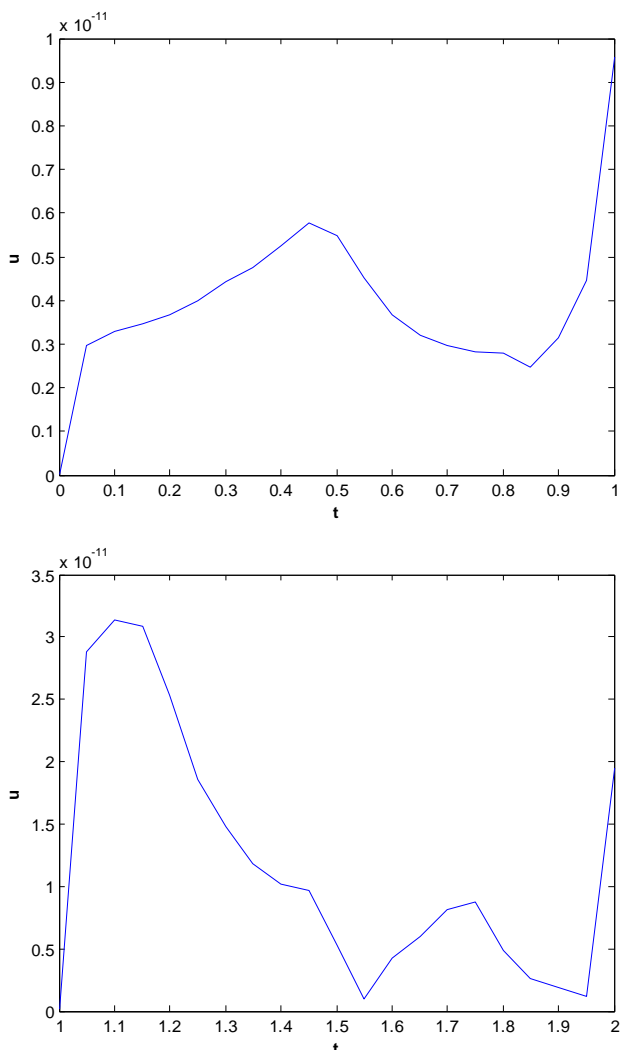

Fig. 4. Relatively errors between the LWPIM and the exact solution of Exp.3.

\section{CONCLUSION}

In this work, we carefully propose a new Legendre wavelets-Picard iteration method (LWPIM) to handle nonlinear IVPs. The work implements Legendre wavelets to approximate all terms within the framework of Picard iteration method. There are three important points to make here. First, the use of Legendre wavelets operational matrices of integral and derivative overcome the difficulties arising in calculating complicated symbolic integral and differential and hence minimizes the computational work to a tangible level. Second, the new iterate formula can avoid the solution of a system of algebraic equations. Third, the developed vector-matrix form makes LWPIM computationally efficient, in which the existing fast numerical algorithms could be employed to accelerate the process of solution.

\section{ACKNOWLEDGMENT}

This work is supported by National Natural Science Foundation of China (Grant Nos. 41105063). 


\section{REFERENCES}

[1] J. I. Ramos, "Picard's iterative method for nonlinear advection-reaction-diffusion equations," Applied Mathematics and Computation, vol. 215, pp. 1526-1536, 2009.

[2] J. I. Ramos, "On the Picard-Lindelof method for nonlinear second-order differential equations," Applied Mathematics and Computation, vol. 203, 2008, pp. 238-242.

[3] G. Beylkin, R. Coifman, and V. Rokhlin, "Fast wavelet transforms and numerical algorithms," I, Commun. Pure Appl. Math, vol. 44, 1991, pp. 141-183.

[4] F. Mohammadi and M. M. Hosseini, "A new Legendre wavelet operational matrix of derivative and its applications in solving the singular ordinary differential equations," J. Franklin Inst., vol. 348, no. 8, 2011, pp. 1787-1796.

[5] U. Lepik, "Numerical solution of evolution equations by the Haar wavelet method," Applied Mathematics and Computation, vol. 185, 2007, pp. 695-704

[6] S. G. Venkatesh, S. K. Ayyaswamy, and S. Raja Balachandar, "The Legendre wavelet method for solving initial value problems of Bratu-type," Computers and Mathematics with Applications, vol. 63, 2012, pp. 1287-1295.

[7] S. A. Yousefi, "Legendre wavelets method for solving differential equations of Lane-Emden type," Applied Mathematics and Computation, vol. 181, 2006, pp. 1417-1422.

[8] R. K. Pandey, N. Kumar, A. Bhardwaj, and G. Dutta, "Solution of Lane-Emden type equations using Legendre operational matrix of differentiation," Applied Mathematics and Computation, vol. 218, 2012, pp. 7629-7637.

[9] M. Razzaghi and S. Yousefi, "The Legendre wavelets operational matrix of integration," Int. J. Syst. Sci., vol. 32, 2001, pp. 495-502.

[10] M. Dehghan and F. Shakeri, The numerical Solution of the Second Painlevé Equation.

[11] S. S. Behzadi, "Convergence of Iterative Methods for Solving Painlevé Equation," Applied Mathematical Sciences, vol. 4, 2010, no. 30, pp. 1489-1507.

[12] E. Hesameddini, "The Use of Variational Iteration Method and Homotopy Perturbation Method for Painlevé Equation I," Applied Mathematical Sciences, vol. 3, 2009, no. 38, pp. 1861-1871.

[13] S. Abbasbandy and Iterated, "He's homotopy perturbation method for quadratic Riccati equation," Appl. math. Comput., vol. 175, 2006, pp. 581-589.

[14] S. Abbasbandy, "A new application of He's variational iteration method for quardratic Riccati differential equation by using Adomian's polynomials," J. Comput. Appl. Math., vol. 207, 2007, pp. 59-63.

[15] C.-Q. Yang, J.-H. Hou, and B.-B. Qin, "Numerical solution of Riccati differential equations by using hybrid functions and tau method," International Journal of Mathematical and Computational Sciences, vol. $6,2012$.

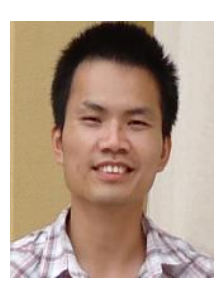

Fukang Yin was born in Guangxi, China, in 1984. He received the bachelor degree in applied mathematics from University of Electronic Science and Technology of China (UESTC) in 2008 and master degree in Computer Science from National University of Defense Technology (NUDT) in 2011. Now he is a PhD candidate student in NUDT. His main research interests are in numerical methods for PDEs and Parallel computation.

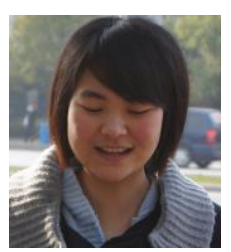

Wangyi Han was born in Shanxi, China, in 1990 She received the bachelor degree in Computer Science from University of Electronic Science and Technology of China (UESTC) in 2010. Now she is an enrolled postgraduate in National University of Defense Technology (NUDT). Her main research interests are in NWP and Parallel computation.

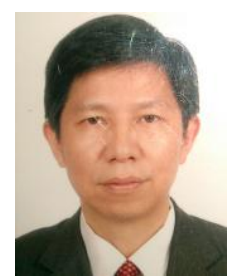

Junqiang Song was born in Hunan, China, in 1962 $\mathrm{He}$ received the bachelor and master degrees in Computer Science from National University of Defense Technology (NUDT) in 2011. Now he is a Professor in NUDT. His main research interests are in Parallel computation, numerical weather prediction, and data assimilation.

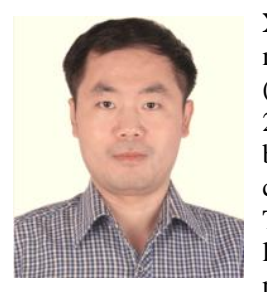

Xiaoqun Cao received the B.S. and Ph.D. degree in meteorology from the People's Liberation Army (PLA) University of Science and Technology in 2002 and 2007, respectively. Since 2007, he has been an Assistant Professor with the school of computer, National University of Defense Technology. His research interests include large-scale scientific computing, numerical weather prediction. 